\title{
Mobile Computing and Cloud maturity - Introducing Machine Learning for ERP Configuration Automation
}

\author{
Elena Geanina ULARU ${ }^{1}$, Florina Camelia PUICAN ${ }^{1}$, George SUCIU ${ }^{2}$, \\ Alexandru VULPE ${ }^{2}$, Gyorgy TODORAN ${ }^{2}$ \\ ${ }^{1} \mathrm{PhD}$ Student, Institute of Doctoral Studies Bucharest \\ ${ }^{2}$ Faculty of Electronics, Telecommunications and Information Technology, University \\ POLITEHNICA of Bucharest, Romania \\ ularugeanina@yahoo.com; puicanflorina@yahoo.com; george@beia.ro; \\ alex.vulpe@radio.pub.ro; todoran.gyorgy@gmail.com
}

Nowadays the smart phone market is clearly growing due to the new type of functionalities that mobile devices have and the role that they play in everyday life. Their utility and benefits rely on the applications that can be installed on the device (the so-called mobile apps). Cloud computing is a way to enhance the world of mobile application by providing disk space and freeing the user of the local storage needs, this way providing cheaper storage, wider accessibility and greater speed for business. In this paper we introduce various aspects of mobile computing and we stress the importance of obtaining cloud maturity by using machine learning for automating configurations of software applications deployed on cloud nodes using the open source application ERP5 and SlapOS, an open source operating system for Decentralized Cloud Computing.

Keywords: Cloud Computing, Mobile Computing, SlapOS, Machine Learning, ERP5

\section{1} Introduction

In our opinion the triggers of today's and future IT market are mobile devices, cloud and social networks. According to Gartner's analysis, "mobile experience is overshadowing the desktop experience [1]." The clientserver architecture is left behind and cloud computing put together with mobile devices draw the modern cloud-client architecture, where the cloud client keeps the usual programming platform and programming language model. From the user experience perspective, cloud combines the benefits of accessing services and having available storage settings. One of the most valuable features of cloud computing is represented by mobility. It is a fact that smartphones are becoming ubiquitous as, in 2012; the smart phone shipment on a global level grew 43 percent reaching 700 million units, according to Research firm Strategy Analytics [2]. Also, according to Credit Suisse, predictions for 2014 estimate one billion devices sold solely in that year and by 2016 there will be at least ten billion smartphones connected to the Internet via mobile apps [3].
Also besides this new feature of Cloud Computing it is important to reach Cloud Maturity. That is why this article describes the methodology to design and implement an open source test platform for machine learning and Enterprise Resource Planning (ERP). In addition, we present an approach to test different machine learning solutions and an example of results obtained by using ERP5. The conclusion summarizes the contributions.

\section{Mobile Applications}

In this chapter we analyze the evolution of mobile development which now raises the expectations from design and development points of view. The mobile operating systems used until now brought their advantages. Among these, there are Android, Blackberry, iOS, Symbian, WebOS, Windows Phone, Bada, Tizen, but the features that meet higher standards are offered today as for example, by Firefox OS, an open source operating system. This operating system is based on a HTML5 web browser and new APIs from Mozilla. HTML5 represents a new framework that enriches the user mobile web expe- 
rience across devices. It represents an intelligent usage of less code to optimize web applications. Users expect to access the published content through a wide range of platforms with a minimum engaged complexity [4].

The mobile evolution from the usual consumer applications to more complex enterprise applications goes toward a single tool that matches all the mobile applications. An issue raised by the content of applications is represented by the request to develop different renditions based on various features of the devices. A common approach would be to have applications built upon open standards, but there is reluctance towards adopting open applications. Native applications offer a package of content and services and they permanently verify what it occurs on their own platforms. These applications put a barrier on what HTML5 web applications are able to accomplish from the technical point of view and business motives. However, HTML5 is not able to adjust to all the features of mobile devices and it is also incapable of replacing on its own what native applications can offer.

Table 1 shows a parallel comparison between HTML5 usage and native applications which would emphasize aspects such as the benefits of the latter:

Table 1. Native Applications vs. HTML5

\begin{tabular}{|c|c|}
\hline $\begin{array}{c}\text { Native applications } \\
\text { Full package of in-applications } \\
\text { and ads }\end{array}$ & Reaches cross platforms \\
\hline Camera & $\begin{array}{c}\text { Encompasses fast and cheap develop- } \\
\text { ment capabilities }\end{array}$ \\
\hline Library & Discoverable research engine \\
\hline Address book & Provides local storage of data \\
\hline $\begin{array}{c}\text { Marketing through application } \\
\text { store }\end{array}$ & $\begin{array}{c}\text { Usage of less code to optimize web ap- } \\
\text { plications }\end{array}$ \\
\hline
\end{tabular}

HTML5 also has disadvantages such as the lack of push messaging, access to camera, library, and address book and there is no distribution available in application stores. Mobile web applications target clients eager to explore and native applications create a relationship with clients based on fidelity.

Overall, HTML5 represents an appreciated way of creating valuable mobile applications. The cross platform tools used for development in mobile applications are centralized on a cloud. The cloud is the platform that allows customers and developers to build and conceive mobile applications. This platform allows the optimization of the services, encompasses analytics and it holds push notifications.

Environments for mobile applications are offered .e.g. by Brightcove App Cloud and Application Craft [5]. These allow end users to customize the applications built using HTML5 in order to be used on different mo- bile operating systems. Brightcove App Cloud is used by developers to even build native applications for iOS and Android. On the market, there is already a competition for cross-platform development tools, the above mentioned platforms being compared with PhoneGap platform [5].

Once the applications are developed and released there is the need of maintenance and further optimization. Brightcove App Cloud enables the support for the whole cycle of mobile applications, on different platforms. App Cloud puts together the native blocks with HTML5 in order for the users to be able to build up, customize and visually design applications without programming knowledge. After the publishing of an application, App Cloud is able to maintain an ongoing optimization in order to enrich the end user experience. All the development tools available, in combination with Cloud func- 
tionalities make the entire development process of mobile applications easier.

App Cloud relies on open standards such as HTML5, JavaScript and CSS3, so the need to know particular programming languages, as for example Java for Android is not necessary. It allows using templates to build different applications. The changes and customizations done for the mobile application are in real-time and they are immediately pushed to the end-users. Using open standards and limiting the complexity of application development, smaller organizations can become capable of launching mobile applications, so the extensive coverage of large organizations is reduced.

Another API alternative for building mobile applications that access the native functionalities of the devices is Apache Cordova. Developing with this API requires the usage of HTML, CSS and JavaScript, without having to use native code, such as C or Java. Apache Cordova's Javascript libraries can be deployed on iOS, Blackberrry, Android, Windows Phone, WebOS, Symbian and Bada so the cross-platform consistency represents again an advantage.

\section{Storage for Mobile Applications}

Mobile applications come with the advantage of data portability. Nevertheless, sometimes, it might happen that the connection to the Internet is not available and this is the crucial moment when mobile web applications are affected. The evolution of mobile development has reached the point where web applications may work offline, without the connection to a network.

One of the reasons why native applications held the monopoly on web applications is the local storage. The operating system of the native applications comes with a layer that allows the storage of data in XML files, INI files or registry, sometimes being possible even to include separate databases as long as the platforms permit.

When analyzing the objectives of web development, we observed that one of them was the data storage on the client side, at first using the HTTP cookies, where the local stor- age of data was possible for a limit of $4 \mathrm{~KB}$ of data. Among the drawbacks of storing data as such was the fact that at each HTTP call, cookies were attached, which leads to situations where data was sent insecure over and over again, unless the web application ran over a SSL (secure sockets layers) connection. SSL is a protocol that ensures a protected communication over the Internet. For a mobile web application to be able to work offline it is necessary to hold large amounts of data on the client side, data that remains available after the page is refreshed and which may or may not be sent to the server. Offline web applications are based on data from HTTP received from the servers. When everything needed to display a page is cached (Javascript, HTML, CSS files), the web pages are loading fast. For offline applications it is imperiously necessary to have everything cached by the browsers. When resources are cached, while the network connection is lost, the web pages are not loaded from the servers; they are accessed from local storage. When connectivity to a network is regained, the cached memory is compared to see if there are any new items. If the items were changed then they are automatically downloaded and uploaded. This way web application are becoming more accessible to users, due to the fact that the time spent for loading pages is reduced by the local storage of the data that was usually retrieved each time from the servers

Nowadays, HTML5 browsers allow the replacement of data in structured key-value local storage. The key-value stored data has a limit size with limits measured in Megabytes, not in Kilobytes as it is the case with cookies. The storage is of two types: local storage and session storage. The server loading side is replaced by client-side usage through clientside scripts that permit local storage of data in a JavaScript database, where data is manipulated using SQL [6].

A JavaScript database class, based on SQLite, provides a relational database intended for local storage of content that is too large to conveniently store in cookies (or is too important to risk accidentally deleting 
when the user clears out his or her cookies) [19].

Storage on different mobile device platforms is available by using for example Cordova API [7].

Web databases are supported by Opera Mobile, Safari Mobile and Chrome for Android web browsers. The databases can be created by APIs, and they can also be opened and closed via APIs. There are some essential methods used to manipulate data for mobile databases: openDatabase, transaction and executesql [19].

In order to create database the openDatabase method is used and the output is a Database object, built through a SQLite Database. The Database object is further used in order to manage data. This database is created on the HTML page and its population is not done as usual via data input. The supported platforms are Android, iPhone, Android, WebOs and Tizen. The method has arguments, such as database version, database_name, database displayname, database size etc. See the following example, where a Database object is obtain from the call of window.openDatabase method [8]:

Var database = window.openDatabase ("trial", "1.0", "New Database", 2000)

The objects used by Cordova are: Database, SQLTransaction, SQLResultset, SQLError and localstorage. The local storage is not intended for the storage of a large amount of data. For localstorage objects, there are methods defined such as localstorage.key aimed to have as output an index, localstorage.getItem - to identify a value of the particular argument, localstorage.setItem - keeps the argument and the value, localstorage.removeItem deletes the value of the particular argument and localstorage.clear - deletes the content storage from localstorage. [8]

The disk space for the databases should be defined by the users and there should be implemented warnings for quota limits. Also, the permission to modify the space used should be available. When using Opera, the database is created without a quota warning for the database size. Nevertheless, in Safari the user receives a warning if the created database has a size higher than the default size, which is $5 \mathrm{MB}$.

After we assessed the evolution of mobile development and the advantages of using the latest technologies we can conclude that flexibility is easier than ever to be gained. Mobile applications are becoming more interactive and display their content in a more efficient way. [9] Now applications are available in offline mode due to local storage and users can access information despite the missing network connectivity. This is all possible due to the offline databases concept within HTML5 from the client side perspective.

\section{Mobile Cloud and the Enterprise}

Usually new technologies are first adopted by big enterprises and after this adoption, they come to the masses. This is the way it happened with cloud computing. It was developed as a concept in order to improve business activity and after that it was extended to the personal use. Nowadays you can have your space in the cloud where you store your files by using different cloud service providers. The situation is different with mobile devices. At the beginning, mobile devices were used to access applications and also information in the cloud but nowadays they are extended to the enterprise as a way of doing business on the move.

As we previously mentioned we consider mobile devices and cloud technology the main drivers for the new IT market, especially on the business level and mobility is the main factor leading to our beliefs.

The definition provided by the Mobile Cloud Computing Forum [10] considers mobile computing as referring to "an infrastructure where both the data storage and the data processing happen outside of the mobile device. Mobile cloud applications move the computing power and data storage away from mobile phones and into the cloud, bringing applications and mobile computing to not just smart phone users but a much broader range of mobile subscribers".

Cloud computing can be defined as "a solu- 
tion that provides new ways of using external resources that you can configure according to your needs - servers, storage, applications and services. A great economical advantage is that resources are leased by the Cloud provider on-demand and in variable quantities so that the client only pays what he consumes. In other words, if you do not use the resources, you do not pay it."[11] The characteristics comprised in this definition together with the portability and flexibility of mobile devices creates the possibility to transform any enterprise to an enterprise in motion. The motion is represented by the enterprise's connectivity to every employee, customer and partner in a fast and cost effective manner. What we have observed during this study is that because the mobile devices were firstly designed for personal use there is little extension to the enterprise level. The cloud today doesn't take explicitly into consideration mobile applications. That is why important changes have to be made in order to ease mobile computing adoption.

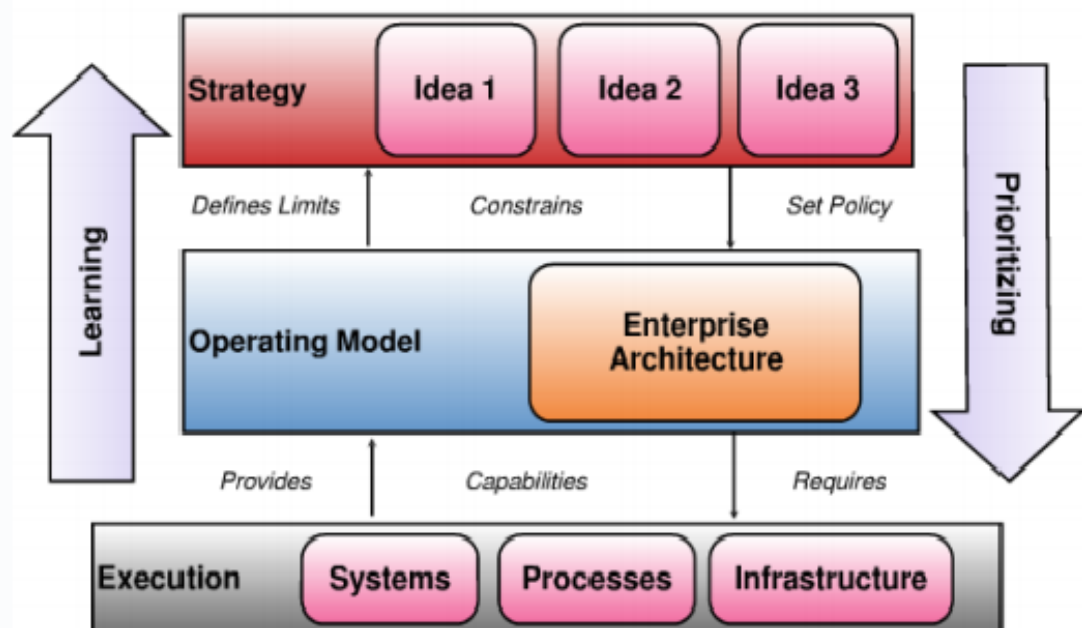

Fig. 1. Including Mobile computing in the Enterprise

At the beginnings of Cloud Computing there was stress on the importance of standards in order to survive as a technology not only a buzz word and so these standards were developed step by step as a sign of market maturity. In our opinion this maturity was reached too early due to the ongoing potential of cloud technology. This potential was demonstrated by the need to integrate mobile computing. The existing standards, although not unified, do not support mobile computing thus they need to be rethought.

In order to successfully adopt mobile computing, companies need to align their Business Strategy and Enterprise Architecture to the new technology.

As we can see in Figure 1, in order to add a new idea to the strategy plan, like moving towards mobile computing you need to have an enterprise architecture that allows systems, processes and infrastructure to be ready for the change.

\section{Cloud Computing and the Enterprise}

We consider Mobile computing as the future and an innovation to cloud computing but we do realize the importance of Cloud as the basis for every other development. That is why this article takes also Machine Learning and Data Mining in the cloud into consideration as a step towards cloud maturity.

Once a company uses an open source system in the cloud to manage everyday information, it is important to learn how to use such information to help with decisions which have to be made. Creating good reports thanks to Machine Learning and Data Mining tools is a very important aspect of the ERP/CRM/DMS systems. Here we introduce the open source software ERP5 and compare it with different open source solutions for machine learning and data mining.

We will introduce in this article SlapOS, an open source operating system for Decentralized Cloud Computing. SlapOS is based on 
the following daemons: buildout - to build any application from source code or binaries located in package repositories, supervisord to monitor and control the processes of the instantiated software and, finally, slapgrid, that implements the decentralized cloud protocol and accounting functions. [12]. SlapOS is based on a master-slave architecture, where the master has the scheduling and accounting role, thus sending status requests and collecting process resource usage from slave nodes. The SlapOS kernel is built on the ERP5 cloud engine model to handle the billing and resource optimization. SLAP stands for "Simple Language for Accounting and Provisioning".

This structure has been implemented for cloud-based automation of ERP and Customer Relationship Management (CRM) software for small and medium enterprises (SMEs) and aspects are under development under the framework of the European research project "Cloud Consulting" [13].

ERP systems are at the core point of a SMEs management and reporting activities. Also SMEs can compete with large enterprises by employing similar technologies in the cloud, that have been available only for organizations that could afford paying consulting services and maintaining such costly infrastructures [14].

Here we use the answers provided by a Chief Executive Officer (CEO) of a SMB in the form of a questionnaire and deploy Machine Learning and Data Mining methodologies to automatically generate an initial basic configuration. The initial configuration is applied to the ERP5 system that is hosted as Software as a Service (SaaS) on a public or private cloud. Furthermore, the configuration can be refined by human IT consultants, and result in a functional configuration. In [13] the authors have presented the applicability of these approaches to automate ERP configuration for SMEs and use open source distributed cloud systems for hosting this type of web-based application.

This approach will significantly reduce time and cost of ERP implementation for SMEs. A CEO of a small business can spend just one hour of his time to answer twenty or thirty questions resulting in a made-to-measure ERP to meet most explicit and implicit requirements of his company.

Also in this paper we present a first prototype application of machine learning for configuring an ERP. This technological breakthrough was not possible before the advent of Cloud Computing and SaaS. In previous approaches ERPs were implemented by consultants, in most cases on a local server. No company could aggregate enough ERP user cases to consider automating the configuration process and providing the necessary resilience [15].

\section{Machine Learning Analyses and Meth- odology}

The current research is based on a designscience approach [16] that has its origins in engineering, and can be considered a problem solving approach aiming to create innovative and useful IT artifacts. The following IT artifacts can be defined:

- Constructs (vocabulary and symbols),

- Models (abstractions and representations),

- Methods (algorithms and practices),

- Instantiations (implemented and prototype systems).

In our project we use the latter three artifacts and an instantiation (a prototype) is considered to be the final outcome. Design-science research can be based on explicit frameworks which provide a set of guidelines for conducting and evaluating good design-science research.

We use the SlapOS platform implemented during the "Cloud Consulting" project [13] hosted on several servers running Ubuntu Linux - Apache - MySQL with current software release. On our cloud testing environment we provide the platform for the installation of thousands of different companies, enabling the analysis of ERP usage data through a large sample of companies.

In the case of our research the most important input artifacts are SlapOS and the underlying ERP5 and the data mining tools offered by Rapid-I [14]. All were assessed as 
suited. Within the overall design science project other research methods are embedded. These are expert interviews to capture human configuration knowledge, multiple case studies to feed the data mining parts with configuration data and to assess the outcome of the automatic configuration, prototyping to test for technical feasibility in detail. This makes our technological approach applicable also to automated provisioning of cloud services [17] and ready to be disseminated much quicker.

First step will be modeling an enterprise based on a collection of questions, possibly several hundred questions. These questions will be the basis for the development of expert knowledge and machine learning models. They will also be used to collect sample data for the data mining approach.

Next step will be creating knowledge engineering technologies and models based on explicit rules which do not require preexisting sample data and which can produce a rough configuration. Such models will be used to reduce the number of questions an SME CEO will need to answer, and to generate default configuration values and we also use data mining to optimize rules.

Third step is creating machine learning models based on data mining with sample data which can be used to optimize the models. Also, some statistical models can be generated to improve the CEOs' replies by analyzing the evolution of the ERP configuration.

\section{Automation Procedure and Information Sources}

To follow the steps described in the methodology, data is gathered from different information sources that belong to different knowledge domains, as show in Figure 2 [18].

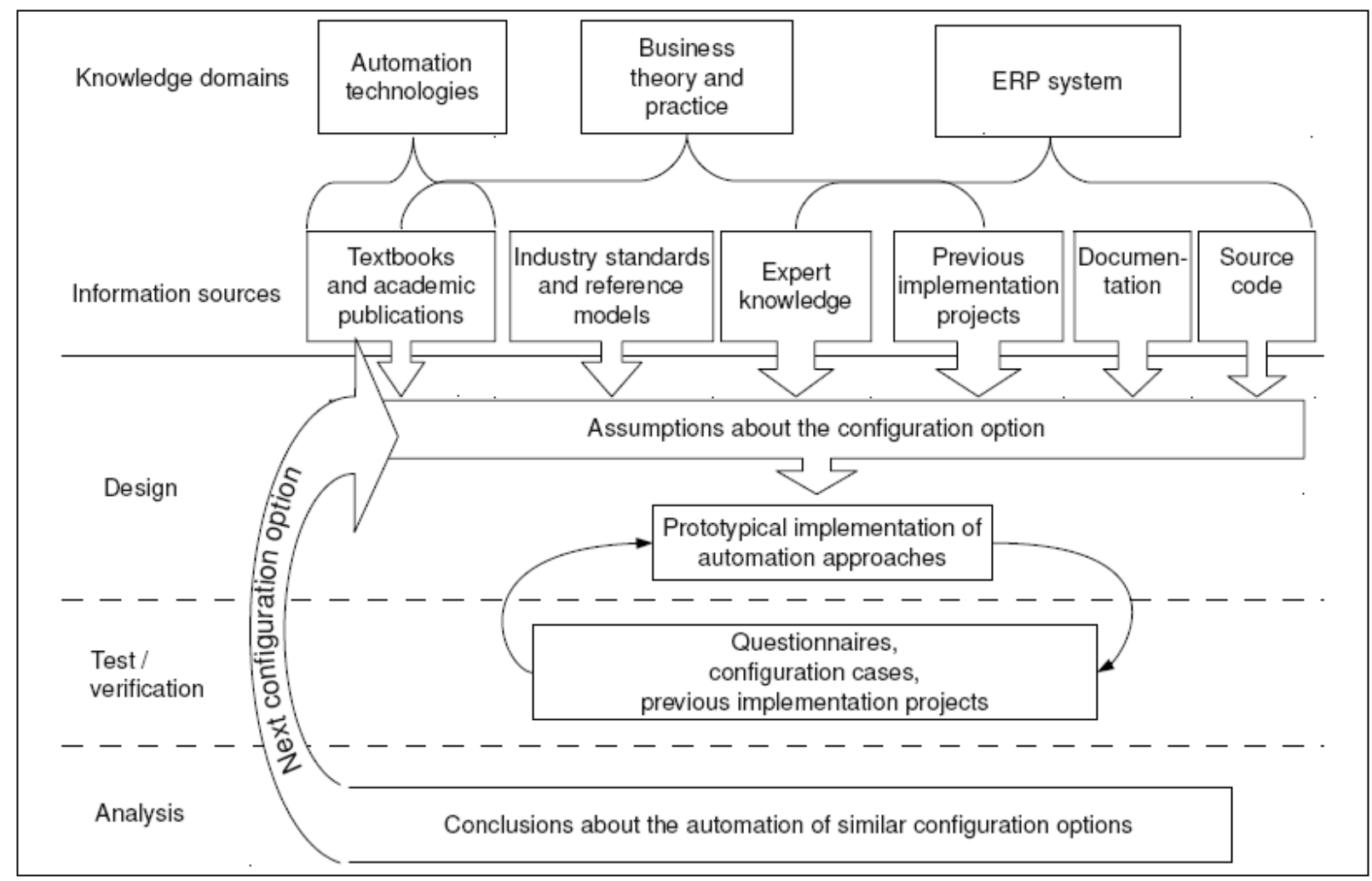

Fig. 2. Proposed automation procedure

By using the flexible database structure of ERP5, where tables can be configured and added to the catalog without the need to interrupt the data warehouse, we can reconfig- ure the system structure according to the configuration values.

The accepted configuration values have as origin the knowledge and assumptions about 
the current ERP system. The effect of the automated configuration options are analyzed and tested, so that the conclusions of the automation can be applied also to similar configuration scenarios [18].

The following information sources are considered when making assumptions about the configuration options:

- Textbooks and academic publications,

- Industry standards and reference models,

- Expert knowledge, including activity reports of developers and consultants who implement the ERP systems,

- Previous implementation projects, including configuration values and documentation,

- Documentation, such as design and architectural documents, technical and implementation documents, user manual

- Source code and screen shots

The most difficult information source to gather is the expert knowledge on category implementation [18]. This can be done by:

- Conducting and initial interview with experts on the various components of the ERP system,

- Questionnaire to be completed by expert stakeholders so that the initial assumptions can be verified against the prototype implementation,

- Knowledge generated by the automation procedure itself,

- Interactions with ERP implementation stakeholders, such as developers, testers and consultants.

Based on the information gathering, analysis and assumptions, one of the available automation approaches is selected and the ERP configuration is automatically implemented in the form of a first prototype.

Furthermore, the initial questionnaires can be used to validate and verify the assumptions on which the prototype is implemented.

\section{Automation Approaches}

We consider two approaches to automate the configuration of categories that are based on artificial intelligence algorithms.

The first approach is based on knowledge engineering and defines a decision tree that models the rules for the categories which will be configured.

The second approach is following a machine learning model where a set of answers and a set of categories are used as training data for a classifier.

As a common point, both approaches are building an expert system where answers to a list of questions are stored. Based on this initial data the category configurations are generated.

The disadvantage of knowledge engineering approach is the scaling of the decision tree, which must be manually defined by an ERP implementation expert with the aid of a knowledge engineering expert. This requires knowledge about the specific ERP system, the technical configuration parameters and options. Also the mapping of the different business requirements to the configuration categories is impossible to be done for all configuration cases. The configuration cases supported by the decision tree have therefore to be narrowed, as seen in Figure 3 [18].

On the other side, the machine learning approach has the problem of automatic category configuration that can be treated as a classification problem. The problem can be considered similar to the problem of text classification where a document has to be classified into categories, and solved such as classifying email into categories "Spam" and "Not Spam". The documents to be classified in this automation approach are sets of answers to a questionnaire. Likewise, a Python script can be used to classify a textual description of the business activity of a company to a set of product line categories [18]. 


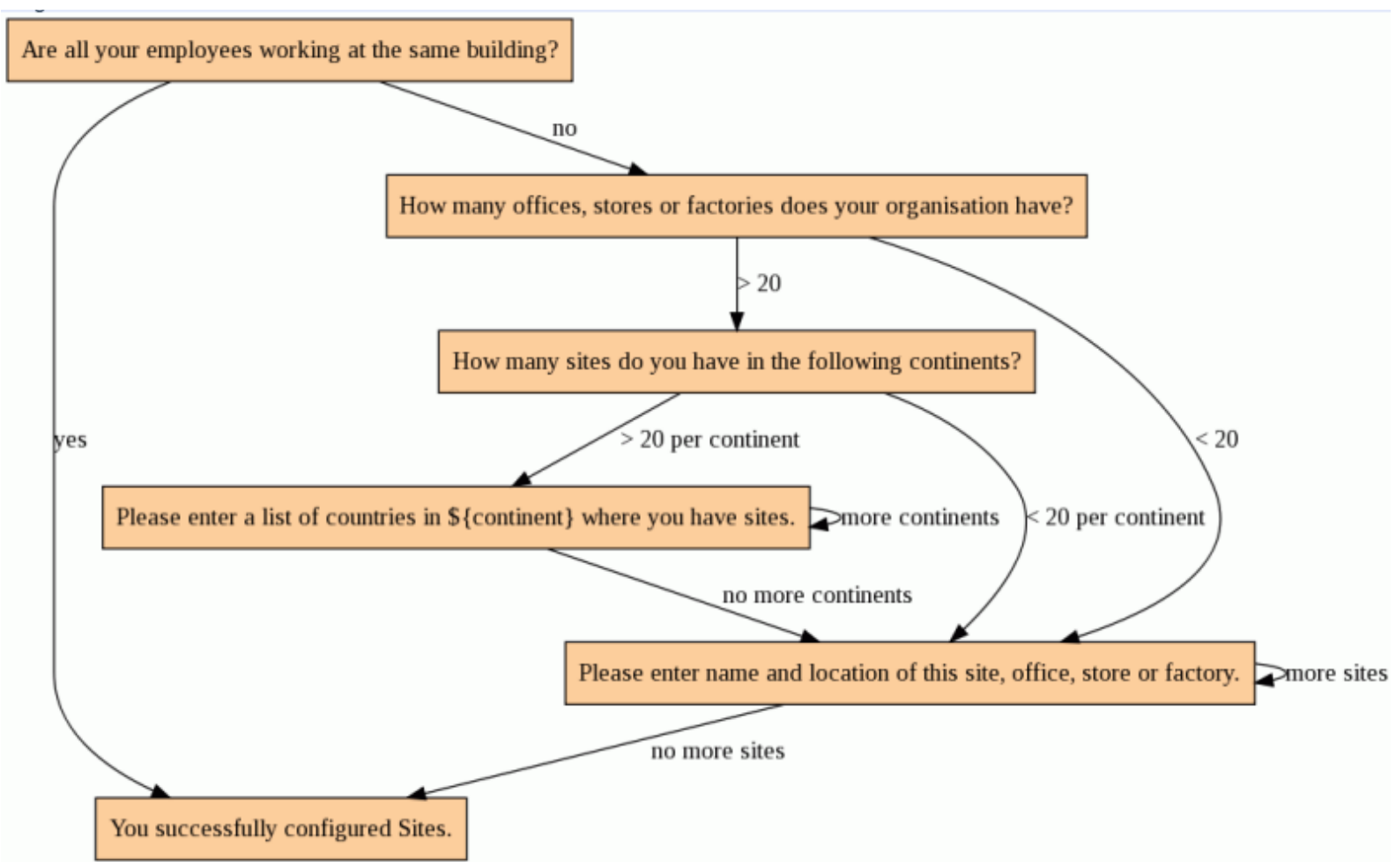

Fig. 3. Decision tree design tool developed in ERP5

The script we first developed in Python defines a list of questions, which are described by the following fields:

- $i d$ : the internal identifier of the question,

- title: the question as it is displayed to the user in the questionnaire system,

- values: a list of possible answers, includ- ing also previously corrected answers,

- type: either "selection" (only a single answer is accepted) or "multiple selection" (multiple answers are accepted).

In our example four questions are defined in the question list:

\{

'id' : 'manufacturing',

'title' : 'Are you a manufacturing organization?',

'values' : (' $\left.\mathrm{y}^{\prime},{ }^{\prime} \mathrm{n}^{\prime}\right)$,

'type' : 'selection',

\}, \# might influence: role, function, activity, publication_section

\{

'id' : 'manufacture-what',

'title' : 'What do you manufacture?',

'values' : ('products', 'spare parts', ),

'type' : 'multiple selection',

\}, \# might influence: product_line, activity, function

\{

'id' : 'manufacture-how',

'title' : 'How do you manufacture your products ?',

'values' : ('oem', 'factory', 'outsource', 'other', ),

'type' : 'multiple_selection',

\}, \# might influence: role, publication_section

\{

'id' : ' supplier-types',

'title' : 'What types of suppliers do you have?',

'values' : ('business', 'not-for-profit', ),

'type' : 'multiple selection',

\}, \# might influence: role, publication_section, product_line, activity 
It is useful to add a commentary at the end of each question that is a rough assumption of which categories could be influenced by the respective question [18]. The initial training

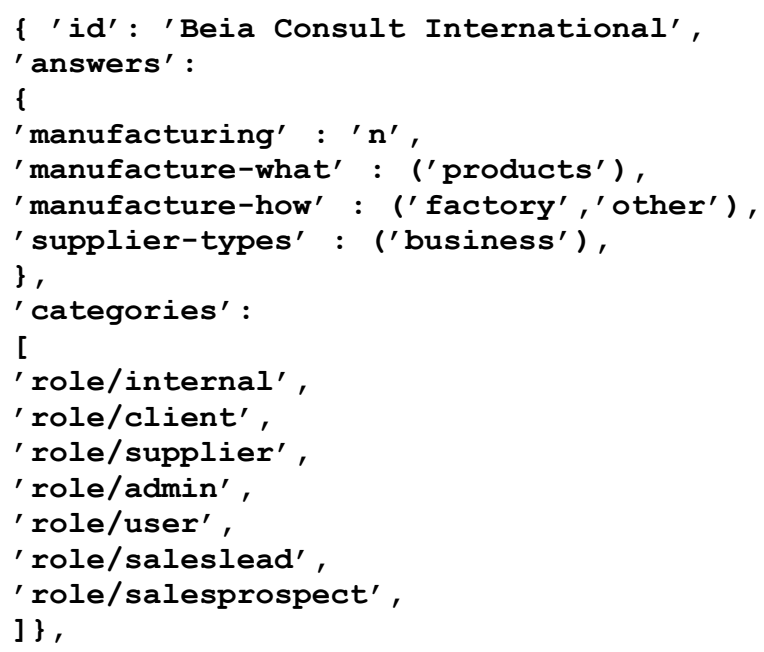

In Figure 4 we show the question management tool we developed in ERP5 design tool for company Beia Consult. The web interface allows creating and collecting the different data in our prototype consists of four examples, each defined by an id, a set of answers and a set of assigned categories, for example: types of configuration questions, such as string (free text, multiple answers) or boolean questions.

\begin{tabular}{|c|c|c|c|c|c|}
\hline View History & \multicolumn{5}{|c|}{ Metadata } \\
\hline \multicolumn{6}{|l|}{ 霍! 嚾 } \\
\hline \multirow[t]{2}{*}{ Title } & \multicolumn{2}{|c|}{ What are your clients? } & Comments & \multicolumn{2}{|c|}{$\begin{array}{l}\text { To be used as input for learning classifier } \\
\text { forl role category. }\end{array}$} \\
\hline & & & Validation State & \multicolumn{2}{|l|}{ Validated } \\
\hline \multicolumn{6}{|l|}{ Description } \\
\hline \multicolumn{6}{|c|}{ Possible Answers: 4 records } \\
\hline \multirow{2}{*}{\multicolumn{2}{|c|}{$\frac{\text { Title }}{\text { Business }}$}} & Value Type & String Value & Integer Value & Float Value \\
\hline & & string_value & business & & \\
\hline \multicolumn{2}{|c|}{ Consumer } & string_value & consumer & & \\
\hline \multicolumn{2}{|c|}{ Not-for-profit Organisations } & string_value & not-for-profit & & \\
\hline \multicolumn{2}{|c|}{ Public administration } & string_value & public_administration & & \\
\hline
\end{tabular}

Fig. 4. Question management tool for machine learning

The questionnaire results show the main advantage of this approach, that it can be used where few training data is available. Furthermore, to be more flexible for new configuration cases, a predictive engine can be envisioned for suggesting new answer values, if none of the predefined answers fits for the selected industry. The questionnaire system allows also storing the history and reviewing the different versions of the completed answer sets, so that consecutive improvements can be used also for training of the machine learning. 


\section{Conclusions}

After we assessed the evolution of mobile development and the advantages of using the latest technologies we can conclude that flexibility is easier than ever to be gained. Also we demonstrate that machine learning, including knowledge engineering and data mining approaches, are well-developed and mature technologies that can be applied to ERP configuration and their power is illustrated by a growing number of successful applications. Thus, automating configuration will lessen the burden of the implementation process and make ERP more accessible for small and medium sized companies.

In this article we evaluated the importance of the mobile movement and we introduced a new perspective over machine learning in cloud.

\section{Acknowledgement}

This work has been funded by the project "Valorificarea capitalului uman din cercetare prin burse doctorale (ValueDoc)" Proiect cofinantat din Fondul Social European prin POSDRU, contract de finantare POSDRU/107/1.5/S/76909, by the Sectoral Operational Programme Human Resources Development 2007-2013 of the Romanian Ministry of Labour, Family and Social Protection through the Financial Agreement POSDRU/107/1.5/S/76903 and by the project "Cloud Consulting".

\section{References}

[1] Mobile Development, Social Media and Cloud Computing Disrupting IT, Gartner Symposium ITxpo, 21 - 25 October 2012 Orlando, Florida [STUDY] http://www.gartner.com/technology/symp osium/orlando/

[2] Smartphone sales in 2012: Winners and losers, http://articles.timesofindia. indiatimes. com/2013-01-26/telecom/ 36563338_1_smartphone-shipmentsglobal-smartphone-smartphone-sales .

[3] Smartphone Sales to Hit 1 Billion in 2014 [STUDY], http://mashable.com/ 2012/04/12/smartphone-sales-to-hit-1billion/.
[4] F. C. Puican, A. Munteanu, "Mobile Analytics - Modern Decision Support Systems," The 19th International Economic Conference - IECS 2012, Sibiu, 15 iunie 2012, Revista Economica, Revista de teorie si practica economico-financiara, Cod CNCSIS 478 B+, I SSN 1582-6260

[5] Build hybrid native apps for iOS \& Android using HTML5, JavaScript, and CSS, https://appcloud.brightcove.com/

[6] Apache Cordova Documentation, http://docs.phonegap.com/en/2.3.0/cordo va storage storage.md.html

[7] Creating mobile Web applications with HTML 5, Part 2: Unlock local storage for mobile Web applications with HTML 5, http://www.ibm.com/developerworks/xm 1/library/ x-html5mobile2/

[8] HTML5 Web Storage - Cookies Are So 1994! http://blog.newrelic.com/ 2012/09/18/ html5-web-storage-cookiesare-so-1994/

[9] F. C. Puican, A. Munteanu, E.-G. Ularu, "Mobile Data Management For Business Intelligence In Distributed Environment," The 19th International Economic Conference - IECS 2012, Sibiu, 15 iunie 2012, Revista Economica, Revista de teorie si practica economico-financiara, Cod CNCSIS 478 B+, ISSN 1582-6260

[10] Cloud Computing World Forum. The Business of Cloud Explained. http://www.mobilecloudcomputingforum. com [accessed on 25 Jan 2013]

[11] G. Ularu, F. Puican, M. Velicanu, "Analysis on Cloud Computing Database in Cloud Environment - Concept and Adoption Paradigm" Database Systems Journal, vol. III, no. 2/2012, pp. 41-48 http://dbjournal.ro/archive/8/8_5.pdf.

[12] P. Takako Endo, A. Vitor de Almeida Palhares, N. Nunes Pereira, G. Estácio Gonçalves, D. Sadok, J. Kelner, B. Melander, J.-E. Mångs, "Resource Allocation for Distributed Cloud: Concepts and Research Challenges", IEEE Network, Vol. 25, no. 4, pp. 42-46, 2011

[13] G. Suciu, O. Fratu, S. Halunga, C. G. Cernat, V. A. Poenaru, V. Suciu, "Cloud Consulting: ERP and Communication 
Application Integration in Open Source Cloud Systems", 19th Telecommunications Forum - TELFOR, IEEE Communications Society, pp. 578-581, 2011

[14] K. Wölfel, J.-P. Smets, S. Strahringer,: "Automating ERP Package Configuration for Small Businesses". Proc. of IFIP International Conference on Research and Practical Issues of Enterprise Information Systems, Confenis, Natal/Brasil, pp. 25-27, August 2010

[15] G. Suciu, C. Cernat, G. Todoran, V. Suciu, V. Poenaru, T. Militaru, and S. Halunga, "A solution for implementing resilience in open source Cloud platforms", 9th International Conference on Communications, IEEE Communications Society, pp. 335-338, June 2012

[16] A. Gräning, R. Wendler, C. Leyh \&
S. Strahringer, "Rigorous Selection of Input Artifacts in Design Science Research - TAVIAS" Proceedings of 16th Americas Conference on Information Systems, AMCIS 2010, pp. 12-15, August 2010

[17] J. Kirschnick, J. M. Alcaraz Calero, L. Wilcock, N. Edwards, "Toward an Architecture for the Automated Provisioning of Cloud Services", IEEE Communications Magazine, Vol. 48, no. 12, pp. 124-131, 2010

[18] K. Wölfel, "Automating ERP Package Configuration for Small Businesses", 2010.

[19]http://developer.apple.com/library/safari/ \#documentation/iPhone/Conceptual/ SafariJSDatabase-Guide/Usingthe JavascriptDatabase/UsingtheJavascript Database.html

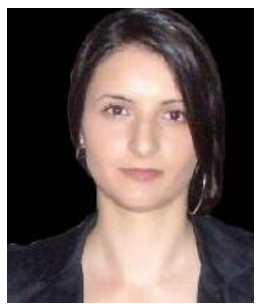

Elena-Geanina ULARU graduated from the Faculty of Cybernetics, Statistics and Economic Informatics of the Academy of Economic Studies in 2008. She holds a Master Degree obtained at Faculty of Cybernetics, Statistics and Economic Informatics of the Academy of Economic Studies at the Academy of Economic Studies and is currently a PhD Student, in the second year, at the Institute of Doctoral Studies, doing her research at the University of Economics from Prague.

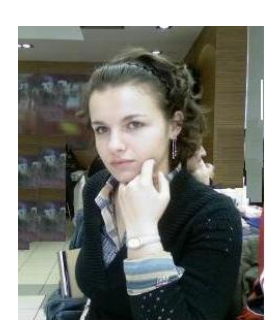

Florina Camelia PUICAN is a PhD Student, in the second year, at the Institute of Doctoral Studies. Bucharest. In 2008, she graduated from Faculty of Business Administration with teaching in foreign languages (English), at the Academy of Economic Studies, Bucharest and in 2009, from Faculty of Mathematics and Computer Science, section Computer Science, University of Bucharest. From 2010, she holds a Master Degree obtained at Faculty of Business Administration with teaching in foreign language (English), at the Academy of Economic Studies, Bucharest. During her studies and work experience she undertook a wide range of skills in economics, information technology and information systems for business, design and management of information systems and databases.

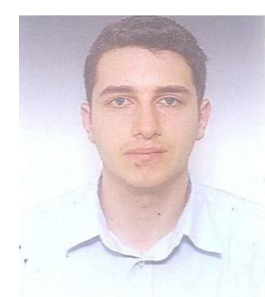

George SUCIU graduated from the Faculty of Electronics, Telecommunications and Information Technology at the University "Politehnica" of Bucharest in 2004. He holds a Master diploma in Informatics Project Management from the Faculty of Cybernetics, Statistics and Economic Informatics of the Academy of Economic Studies Bucharest from 2010 and currently, his $\mathrm{PhD}$ work is focused on the field of Electronics Engineering and Cloud Communications. Also he is IEEE member and has received a type D IPMA certification in project management from Romanian Project Management Association / IPMA partner organization. He is the author or co-author of over 30 journal articles and scientific papers at conferences. His scientific fields of interest include: project management, electronics and 
telecommunication, cloud computing, big data, open source, IT security, data acquisition and signal processing. Known languages: German, French, English; He also has experience in project leading and participation in various research projects (FP7, National Structural Funds), with more than 15 years activity in information and telecommunication systems.

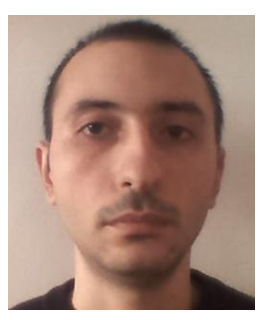

Alexandru VULPE received his B.Sc, M.Sc from the Faculty of Electronics, Telecommunications and Information Technology at the University "Politehnica" of Bucharest in 2009 and 2011, with both theses focused on interoperability between wireless access networks based on the IEEE 802.21 standard, which included software modeling in the $\mathrm{C}++$ language. His main interests are focused on wireless access technologies, integrated telecommunication networks, optimization techniques, software development, mobile application development and his $\mathrm{PhD}$ work is in the area of heterogeneous wireless networks, $4 \mathrm{G}$ and beyond $4 \mathrm{G}$ networks. He is also a participant in several research projects focused on wireless sensor networks (SaRaT-IWSN, CORONA), with an activity related to wireless communications protocols development, simulations and testing and in test applications development.

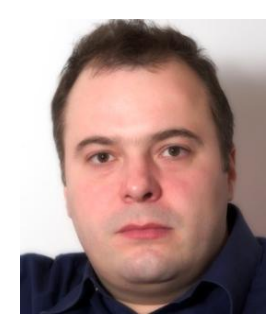

Gyorgy TODORAN has graduated the Faculty of Electronics, Telecommunications at University "Politehnica" in Bucharest in 2000. He holds a Master degree in Quality Management (2001) and Strategic Management (2002) from the "Politehnica" University of Bucharest. Currently he is working on his $\mathrm{PhD}$ thesis in security technologies with focus on open source, cloud computing, mobile and BYOD initiatives. He has more than 10 years experience in commercial and governmental telecommunication systems, mainly in system administration, system management, design, project management, consulting 